\title{
Locoregional Recurrence via Mucus-Mediated Extension Following Lung Resection for Mucinous Tumors
}

Yo Kawaguchi ( $\sim$ kawaguchi1228@yahoo.co.jp)

Shiga University of Medical Science

Jun Hanaoka

Shiga University of Medical Science

Yasuhiko Ohshio

Shiga University of Medical Science

Keigo Okamoto

Shiga University of Medical Science

Ryosuke Kaku

Shiga University of Medical Science

\section{Kazuki Hayashi}

Shiga University of Medical Science

Takuya Shiratori

Shiga University of Medical Science

Akira Akazawa

Shiga University of Medical Science

Mitsuaki Ishida

Kansai Medical University

\section{Research Article}

Keywords: Pulmonary resection, Locoregional recurrence, Mucinous tumor, Mucus extension,

Posted Date: March 9th, 2021

DOl: https://doi.org/10.21203/rs.3.rs-189233/v1

License: (c) (i) This work is licensed under a Creative Commons Attribution 4.0 International License.

Read Full License 


\section{Abstract \\ Background}

Clinically, locoregional recurrences following mucinous tumor resection are often experienced. However, it is unclear whether mucinous tumors directly affect local recurrence or not, and if so, what the mechanism is. Therefore, we investigated whether mucinous tumors are associated with locoregional recurrence after pulmonary resection and whether mucus extension is a risk factor for locoregional recurrence.

\section{Methods}

The data of 90 patients who underwent pulmonary resection for metastases were reviewed. If mucus was partially or wholly present in the tumor based on macro- or microscopic identification, we assigned the tumor as mucinous. In mucinous tumors, if mucus was identified within the air spaces in the normal lung parenchyma, beyond the edge of the tumor, we assigned the tumor as positive for "mucus extension."

\section{Results}

The 5-year cumulative incidence of locoregional recurrence in patients with mucinous tumors was $80.3 \%$, which was significantly higher than the $15.5 \%$ observed in patients with non-mucinous tumors. Within the mucinous tumor, presence of mucus extension beyond the tumor edge was an independent risk factor for locoregional recurrence after pulmonary resection (hazard ratio, 8.08; $P=0.049$ ).

\section{Conclusion}

During the resection of mucinous cancer, surgeons should maintain sufficient distance from the tumor edge to prevent locoregional recurrences.

\section{Background}

Despite the developments in chemo- and radiotherapy, and treatment with biological agents for patients with pulmonary metastases, surgery remains an important treatment option[1]. Locoregional recurrences following lung resection for metastases are often associated with specific clinicopathological features, such as limited resection, large tumor size, histologic type, and tumor spread through air spaces (STAS) [2-5]. Furthermore, these features are associated with both locoregional recurrences and poor prognosis[3, 4]. Mucinous tumors mainly originate from gastrointestinal cancer[6, 7], pseudomyxoma peritonei[8], lung cancer[9], pancreatic cancer[10], uterine cancer[11], ovarian cancer[12], or kidney cancer[13] and sometimes head and neck cancer[14]. Some mucinous tumors also have poor prognosis following surgery $[6,7,9,12]$. 
Clinically, in our hospital, we often observe locoregional recurrences after macroscopic complete resection of mucinous tumors. In addition, we have noticed that mucus derived from the tumor often extends through air spaces into the lung parenchyma adjacent to the tumor edge. We have named this phenomenon "mucus extension." Mucus extension may be important because tumor cells can spread through mucus-mediated extension[8]. However, it is unclear whether this histology actually develop tumor recurrence following pulmonary resection or not. Therefore, using the data of a cohort of patients with resected pulmonary metastases, we investigated whether mucinous cancer is associated with locoregional recurrence following pulmonary resection and whether mucus extension is a risk factor for locoregional recurrence according to the type of surgical procedure.

\section{Methods}

\section{Patient cohort}

We obtained institutional review board approval (chairman: Sueyoshi Moritani) of the Kusatsu General Hospital for this study (Number: 2019-015). The requirement for informed patient consent was waived because of the retrospective nature of the study. All methods were carried out in accordance with Journal's guidelines.

The present study was a retrospective analysis of the medical records of 90 patients who underwent pulmonary resection for metastases at our hospital between September 2007 and December 2019. Patients who met the following criteria underwent surgery: 1) the primary tumor was controlled and 2) there was no effective therapy other than surgery. We defined the primary tumor as "controlled" when the tumor had been surgically resected and there were no recurrences at the time of pulmonary resection. Furthermore, we enrolled patients whose pulmonary metastases could have been macroscopically resected. The medical record of each patient was reviewed for age, sex, primary tumor, tumor size, number of pulmonary lesions, regions of pulmonary nodules and the surgical procedure for the metastasectomy. As a rule, we performed wedge resection for metastatic lesions, using an automated suturing device (Endo GIA tri-stapler, Covidien or Endopath stapler Echeron flex, Ethicon, Cincinnati, $\mathrm{OH}$ ). During the resection, we decided to cut the lung at approximately $1 \mathrm{~cm}$ distance from the tumor edge. We selected segmentectomy, lobectomy, or pneumonectomy if the tumors were located in the hilum or if multiple nodules were present in the same lobe. Following metastasectomy patients were followed-up for a maximum of 5 years. All recurrences were confirmed by radiological assessment. Locoregional recurrence was defined as a nodule occurring on the resection stump of the lung or bronchus and the nodule expanding during follow-up computed tomography.

We classified patients into two groups: a mucinous tumor group and a non-mucinous tumor group. If mucus was partially or wholly present in the tumor based on macro- or microscopic identification, we assigned the tumor as mucinous. We analyzed recurrence-free survival and the cumulative incidence of locoregional recurrence in the mucinous tumor group and compared these values with a control, nonmucinous tumor group. If there were multiple lesions in a single patient, we judged that recurrence had 
occurred if at least one lesion recurred. Furthermore, in the mucinous tumor group, primary tumor, tumor size, regions of pulmonary nodules, surgical procedure, and presence of mucus extension were recorded.

\section{Histologic evaluation}

The surgically resected specimens were fixed in 10\% formalin and cut into 5-10 mm slices. All sections that contained both tumor tissue and surrounding normal lung tissue were embedded in paraffin. Additional $5 \mu \mathrm{m}$ sections were cut from a selected tissue block and stained with hematoxylin and eosin.

We microscopically examined the edge of the tumor or mucus. Specimen sections were divided into two groups: those where the border between the tumor and normal lung tissue was clear (Fig. 1A, 1B) and those where the border was not clear (Fig. 1C, 1D). In the former group, the tumor edge was identified as a smooth surface easily recognizable at gross or low-power field examination, as indicated by the dotted line in Fig. 1A and Fig. 1B. In the latter group, mucus was identified within air spaces in the normal lung parenchyma beyond the edge of the tumor (indicated by arrows in Fig. 1C, 1D). We named this phenomenon "mucus extension." Even if mucus only existed in the first alveolar layer from the tumor edge, mucus extension was considered present. The above pathological diagnoses were performed by Y.K, and one expert pathologist (M.I) checked the diagnoses.

\section{Statistical analysis}

Statistical analysis was performed using SPSS Statistics for Windows, version 25 (IBM Corp., Armonk, NY). Associations between variables were analyzed using Fisher's exact test (for categorical variables) and the Wilcoxon test (for continuous variables). The Kaplan-Meier method was used to determine overall survival, relapse-free survival, and cumulative incidence of locoregional recurrence (CIR). The logrank test was used to compare survival differences for each variable. Cox's proportional hazards model was used for multivariate analysis. Statistically significant differences were defined as $P<0.05$.

\section{Results}

\section{Survival comparison between mucinous and non-mucinous tumors}

To examine the association between mucinous tumors and locoregional recurrence, we compared recurrence-free survival between patients with mucinous and non-mucinous tumors who underwent pulmonary resection for metastases. The characteristics of both groups are shown in Table 1. Twenty-six patients were diagnosed with a mucinous tumor, where the primary tumors were gastrointestinal cancer (54\%), pseudomyxoma peritonei (38\%), lung cancer (4\%), and urinary tract cancer (4\%). Sixty-four patients were diagnosed with a non-mucinous tumor, where the primary tumors were gastrointestinal cancer (64\%), head and neck cancer (11\%), kidney/urinary tract cancer (9\%), uterine cancer (9\%), lung cancer $(2 \%)$, and cancer in other sites (5\%). The pathological types of tumors, including the number of patients and the number of metastatic lesions, are shown in Table 2. The mucinous tumors comprised 
adenocarcinoma in 25 patients (96\%) and clear cell carcinoma in one patient (4\%). The non-mucinous tumors comprised adenocarcinoma in 47 patients (73\%), squamous cell carcinoma in five patients (8\%), clear cell carcinoma in four patients $(6 \%)$, and other types in eight patients $(13 \%)$. The recurrence-free survival after pulmonary resection in the patients with mucinous tumors was $13.6 \%$, which was significantly lower than the $36.7 \%$ observed in patients with non-mucinous tumors (Fig. 2). We suspected that this low recurrence-free survival in patients with mucinous tumors was mainly the result of locoregional recurrences. We therefore examined the CIR and found that the 5-year CIR in patients with mucinous tumors was $80.3 \%$, which was significantly higher than the $15.5 \%$ observed in patients with non-mucinous tumors (Fig. 3).

Table 1

Characteristics of patients with mucinous and non-mucinous tumors.

\begin{tabular}{|c|c|c|c|c|c|}
\hline \multirow[t]{2}{*}{ Variable } & \multicolumn{2}{|c|}{ Mucinous } & \multicolumn{2}{|c|}{ Non-mucinous } & \multirow[t]{2}{*}{$P$ value } \\
\hline & $N=26$ & $\%$ & $N=64$ & $\%$ & \\
\hline \multicolumn{6}{|l|}{ Age, years } \\
\hline Median & 59.5 & - & 64.5 & - & 0.082 \\
\hline Range & $33-77$ & & $29-85$ & & \\
\hline \multicolumn{6}{|l|}{ Gender } \\
\hline Male & 13 & 50 & 35 & 55 & 0.816 \\
\hline Female & 13 & 50 & 29 & 45 & \\
\hline \multicolumn{6}{|l|}{ Primary tumor } \\
\hline Gastrointestinal cancer & 14 & 54 & 41 & 64 & 0.368 \\
\hline Pseudomyxoma peritonei & 10 & 38 & 0 & 0 & $<0.001$ \\
\hline Lung cancer & 1 & 4 & 1 & 2 & 0.505 \\
\hline Kidney, urinary tract cancer & 1 & 4 & 6 & 9 & 0.375 \\
\hline Head and neck cancer & 0 & 0 & 7 & 11 & 0.079 \\
\hline Uterine cancer & 0 & 0 & 6 & 9 & 0.106 \\
\hline Others & 0 & 0 & 3 & 5 & 0.262 \\
\hline
\end{tabular}


Table 2

The pathological types of tumors, and the number of patients and metastatic lesions.

\begin{tabular}{|lllll|}
\hline Variables & \multicolumn{2}{l}{ Mucinous } & \multicolumn{2}{l|}{ Non-mucinous } \\
\cline { 2 - 5 } & Patients & Lesions & Patients & Lesions \\
\hline Total & $\mathbf{2 6}$ & $\mathbf{5 4}$ & $\mathbf{6 4}$ & $\mathbf{1 0 3}$ \\
\hline Gastrointestinal cancer & 14 & 31 & 41 & 60 \\
adenocarcinoma & 14 & 31 & 39 & 58 \\
hepatocellular carcinoma & 0 & 0 & 2 & 2 \\
Pseudomyxoma peritonei & 10 & 21 & 0 & 0 \\
adenocarcinoma & 10 & 21 & 0 & 0 \\
Lung cancer & 1 & 1 & 1 & 1 \\
adenocarcinoma & 1 & 1 & 0 & 0 \\
squamous cell carcinoma & 0 & 0 & 1 & 1 \\
Kidney, urinary tract cancer & 1 & 1 & 6 & 9 \\
clear cell carcinoma & 1 & 1 & 4 & 6 \\
urothelial carcinoma & 0 & 0 & 2 & 3 \\
Head and neck cancer & 0 & 0 & 7 & 18 \\
papillary adenocarcinoma & 0 & 0 & 5 & 15 \\
squamous cell carcinoma & 0 & 0 & 2 & 3 \\
Uterus cancer & 0 & 0 & 6 & 12 \\
leiomyosarcoma & 0 & 0 & 3 & 8 \\
adenocarcinoma & 0 & 0 & 2 & 2 \\
squamous cell carcinoma & 0 & 0 & 1 & 2 \\
The others & 0 & 0 & 3 & 3 \\
adenocarcinoma & 0 & 0 & 1 & 1 \\
adenosquamous cell carcinoma & 0 & 0 & 1 & 1 \\
squamous cell carcinoma & 0 & 0 & 1 & 1 \\
\hline
\end{tabular}

\section{Association between locoregional recurrence and mucus extension}


We suspected that locoregional recurrence would easily occur in mucinous tumors via mucus-mediated extension. We identified 54 metastatic lesions in 26 patients with mucinous tumors, and nine lesions developed locoregional recurrences. Of the 54 lesions, we identified 26 (48\%) with mucus extension present. We extracted the potential clinical risk factors of locoregional recurrence as: maximum tumor size, central region of the tumor (tumor existing partially or wholly inside one-third of the area of a pulmonary pleura), and limited resection (enucleation, wedge resection, or segmentectomy). Associations between these clinical risk factors and mucus extension are analyzed and summarized in Table 3 . The risk of developing locoregional recurrence was significantly higher in patients with tumors in the central region than in the peripheral region (3-year CIR, $75.7 \%$ vs. $5.3 \% ; P=0.029)$ and in patients with mucus extension present than with mucus extension absent (3-year CIR, $100 \%$ vs. $5.6 \% ; P=0.013$ ). In multivariate analysis, presence of mucus extension was the sole independent risk factor for locoregional recurrence (hazard ratio, 8.08; $P=0.049$ ) (Table 4).

Table 3

Clinicopathological associations with locoregional recurrence in mucinous tumors.

\begin{tabular}{|lllll|}
\hline Mucinous tumor variable & Lesions & $\%$ & 3 -year CIR (\%) & $P$ value \\
\hline All lesions & 54 & - & 55.5 & - \\
\hline Maximum tumor size & & & & 0.469 \\
\hline$<20 \mathrm{~mm}$ & 42 & 78 & 28.6 & \\
\hline$\geq 20 \mathrm{~mm}$ & 12 & 22 & 70.8 & \\
\hline Region of the tumor & & & & 0.029 \\
\hline Central & 23 & 43 & 75.7 & \\
\hline Peripheral & 31 & 57 & 5.3 & 0.382 \\
\hline Surgical procedure & & & & \\
\hline Enucleation, wedge, segmentectomy & 49 & 90 & 57.3 & \\
\hline Lobectomy & 5 & 10 & 0.0 & \\
\hline Mucus extension & & & & \\
\hline$(+)$ & 26 & 48 & 100 & \\
\hline$(-)$ & 28 & 52 & 5.6 & \\
\hline
\end{tabular}

Table 4

Multivariate analysis of locoregional recurrence in mucinous tumors.

\begin{tabular}{|llll|}
\hline Variable & HR & $95 \% \mathrm{Cl}$ & $P$ value \\
\hline Region of the tumor: Central & 6.72 & $0.83-54.41$ & 0.074 \\
\hline Mucus extension: $(+)$ & 8.08 & $1.01-64.9$ & 0.049 \\
\hline
\end{tabular}




\section{Limited resection of mucinous tumors and locoregional recurrence}

We considered that limited resection may increase the risk of locoregional recurrence. Of 54 lesions, six were resected using lobectomy, seven using segmentectomy, 40 using wedge resection, and one using enucleation. There was no locoregional recurrence in lesions resected using lobectomy. However, one lesion (14.3\%) resected using segmentectomy developed locoregional recurrence, and eight lesions (19.0\%) resected using wedge resection or enucleation developed locoregional recurrence (Fig. 4).

\section{Discussion}

The purpose of this study was to clarify whether mucinous tumors are associated with locoregional recurrence after pulmonary resection and establish whether mucus extension is a risk factor for locoregional recurrence according to the type of surgical procedure.

Previously, STAS was not accepted as a form of invasion because it is unique to the lungs. Anatomically, the lungs have air pathways, which permit tumor cells to spread. Kadota et al identified STAS in $40 \%$ of lung adenocarcinoma cases and found that locoregional recurrence after pulmonary resection significantly increased in STAS-positive tumors[2]. Shiono et al. also demonstrated that aerogenous spread with floating cancer cell clusters was an independent prognostic factor[4]; moreover, floating cancer cell clusters and a malignant positive surgical margin in the resected specimens carry significantly higher risk for local recurrence[5] in cases of colorectal pulmonary metastasis. Based on these reports, STAS is coming to be recognized as a pattern of invasion. Previously, we showed that mucinous tumors can spread through mucus-mediated extension[8] in a manner resembling dissemination. We, therefore, hypothesized that mucus could easily spread through air spaces and that it might be possible for cancer cells to extend via the mucus, resulting in local recurrence. Here, we demonstrated that recurrence-free survival after pulmonary resection in patients with mucinous tumors was significantly lower than in patients with non-mucinous tumors. This result is similar to those of reports on mucinous tumors found elsewhere in the body, which were also associated with high recurrence rates $[6,7,9,12]$.

We consider that mucus extension could be a sensitive marker of locoregional recurrence similar to STAS. We found mucus extension, identified microscopically based on the lack of a distinct border between the tumor and normal lung tissue, to be present in $48 \%$ of mucinous tumors. We considered that mucus extension might cause tumor spread in these cases and therefore hypothesized that mucus extension might be a risk for locoregional recurrence. Indeed, we found that the 3-year CIR was higher in patients showing mucus extension and that mucus extension was the key independent risk factor for locoregional recurrence following pulmonary resection.

Surgeons need to select the optimal surgical procedure for complete resection of lung tumor. Tumor STAS can be difficult to recognize on the frozen section because STAS tumor cells and alveolar macrophages have similar morphologies. If distinction is difficult, immunohistochemistry for keratin and 
a macrophage marker such as CD68 may be needed[2]. Conversely, mucus extension could be identified using a frozen section during the operation. In this study, we showed that locoregional recurrence occurred in patients who underwent enucleation, wedge resection, and segmentectomy but did not in those who underwent lobectomy, suggesting that limited resection may increase the risk of locoregional recurrence. Therefore, in the future, if mucus extension can be identified during the operation, this may help surgeons decide on the need for additional resection or anatomical lung resection. In this study, locoregional recurrence occurred in cases of mucinous tumors with 1-cm resection margins; therefore, margins larger than $1 \mathrm{~cm}$ from the tumor edge should be selected to avoid recurrence.

The main limitation of this study was the heterogeneity of the primary tumor histology. We resected the metastatic tumors irrespective of their primary histology because a recent report showed the effectiveness of pulmonary metastasectomy[15]. However, tumor characteristics such as growth speed, invasive capacity, and metastatic potential differ both according to mucus existence and according to the histology of the primary lesion. In particular, there was histological variability between patients with mucinous and non-mucinous tumors, which might have affected the recurrence-free survival. A second limitation was the small number of cases, which did not provide sufficient power to detect significant differences; as a result, the statistical may be questionable. Third, we may have underestimated the mucus extension. We observed only the maximum surface of the tumor, and the other surfaces may have potentially contained mucus extension. Finally, the diagnosis of local recurrence was equivocal. We confirmed locoregional recurrence through radiological assessment, not by biopsy, which may sometimes misinterpret inflammatory consolidation as locoregional recurrence.

Based on this original study, we intend to spread awareness of the potential risk of postoperative locoregional recurrence in patients with mucinous tumor and mucus extension. Furthermore, a large trial targeting mucinous tumor resection is required to achieve more precise results in the future.

\section{Conclusions}

We demonstrated that mucus extension may be a risk factor for locoregional recurrence after pulmonary resection for lung metastases. Apart from the histology of the primary tumor, whether the tumor is mucinous or non-mucinous should also be considered when pulmonary resection is planned. In the future, we hope that mucus extension can be identified using frozen sections during surgery and that surgeons will consider additional resection to maintain sufficient distance from the tumor edge and prevent locoregional recurrences.

\section{Abbreviations}

STAS

spread through air spaces; CIR:cumulative incidence of locoregional recurrence

\section{Declarations}


Ethics approval and consent to participate: This study was approved by the institutional review board (chairman: Sueyoshi Moritani) of the Kusatsu General Hospital for this study ((Approval Number: 2019015).the institutional review board. Informed consent was waived by the institutional review board because of the retrospective nature of the study. The data of participants which used in this study was anonymized before its use.

Consent for publication: Not applicable.

Availability of data and materials: The data that support the findings of this study are available from the corresponding author upon reasonable request.

Competing interests: The authors declare that they have no competing interests.

Funding: Not applicable.

\section{Author's contributions}

KY has made substantial contributions to conception and design, or acquisition of data, or analysis and interpretation of data and been involved in drafting the manuscript or revising it critically for important intellectual content. M.I has made substantial contributions to the pathological diagnoses. JH, YO, KO, $\mathrm{RK}, \mathrm{KH}, \mathrm{TS}$ and AA have made substantial contributions to analysis and interpretation of data, and given final approval of the version to be published and agreed to be accountable for all aspects of the work in ensuring that questions related to the accuracy or integrity of any part of the work are appropriately investigated and resolved.

All authors have read and approved the manuscript.

Acknowledgements: None.

\section{References}

1. Kawaguchi Y, Hanaoka J, Ohshio Y, Okamoto K, Kaku R, Hayashi K, Shiratori T, Yoden M: Survival and prognostic factors in patients undergoing extended pulmonary metastasectomy. Mol Clin Oncol 2020, 13(5):48.

2. Kadota K, Nitadori J, Sima CS, Ujiie H, Rizk NP, Jones DR, Adusumilli PS, Travis WD: Tumor Spread through Air Spaces is an Important Pattern of Invasion and Impacts the Frequency and Location of Recurrences after Limited Resection for Small Stage I Lung Adenocarcinomas. J Thorac Oncol 2015, 10(5):806-814.

3. Shiono S, Ishii G, Nagai K, Yoshida J, Nishimura M, Murata Y, Tsuta K, Nishiwaki Y, Kodama T, Ochiai A: Histopathologic prognostic factors in resected colorectal lung metastases. Ann Thorac Surg 2005, 79(1):278-282. 
4. Shiono S, Ishii G, Nagai K, Murata Y, Tsuta K, Nitadori J, Kodama T, Ochiai A: Immunohistochemical prognostic factors in resected colorectal lung metastases using tissue microarray analysis. Eur $\mathrm{J}$ Surg Oncol 2006, 32(3):308-309.

5. Shiono S, Ishii G, Nagai K, Yoshida J, Nishimura M, Murata Y, Tsuta K, Kim YH, Nishiwaki Y, Kodama $\mathrm{T}$ et al: Predictive factors for local recurrence of resected colorectal lung metastases. Ann Thorac Surg 2005, 80(3):1040-1045.

6. Kepil N, Batur S, Goksel S: Immunohistochemical and genetic features of mucinous and signet-ring cell carcinomas of the stomach, colon and rectum: a comparative study. Int J Clin Exp Pathol 2019, 12(9):3483-3491.

7. Lan YT, Chang SC, Yang SH, Lin CC, Wang HS, Jiang JK, Chen WS, Lin TC, Chiou SH, Lin JK: Comparison of clinicopathological characteristics and prognosis between early and late recurrence after curative surgery for colorectal cancer. Am J Surg 2014, 207(6):922-930.

8. Kawaguchi Y, Hanaoka J, Ohshio Y, Okamoto K, Kaku R, Hayashi K, Shiratori T, Yoden M: Patient Survival after Surgical Management in Intrathoracic Pseudomyxoma peritonei. Ann Surg Oncol 2019, 26(1):238-243.

9. Dong Y, Zhou L, Zhao D, Li K, Liu Z, Che N, Liu H: MUC5AC enhances tumor heterogeneity in lung adenocarcinoma with mucin production and is associated with poor prognosis. Jpn J Clin Oncol 2020, 21(5742631).

10. Aronsson L, Bengtsson A, Toren W, Andersson R, Ansari D: Intraductal papillary mucinous carcinoma versus pancreatic ductal adenocarcinoma: A systematic review and meta-analysis. Int J Surg 2019, 71:91-99.

11. Stolnicu S, Hoang L, Soslow RA: Recent advances in invasive adenocarcinoma of the cervix. Virchows Arch 2019, 475(5):537-549.

12. Chen RF, Tao X, Wu BB, Li J, Wang JY, Gu WY, Lu X: Mucinous borderline ovarian tumors with and without Intraepithelial Carcinoma: Differences in clinicopathologic features and fertility results. $J$ Obstet Gynaecol Res 2020, 11(10):14210.

13. Ged Y, Chen YB, Knezevic A, Donoghue MTA, Carlo MI, Lee CH, Feldman DR, Patil S, Hakimi AA, Russo $P$ et al: Mucinous Tubular and Spindle-Cell Carcinoma of the Kidney: Clinical Features, Genomic Profiles, and Treatment Outcomes. Clin Genitourin Cancer 2019, 17(4):268-274.

14. Wang J, Guli QR, Ming XC, Zhou HT, Cui YJ, Jiang YF, Zhang D, Liu Y: Primary mucinous carcinoma of thyroid gland with prominent signet-ring-cell differentiation: a case report and review of the literature: Onco Targets Ther. 2018 Mar 16;11:1521-1528. doi: 10.2147/OTT.S158975. eCollection 2018.

15. Younes RN, Fares AL, Gross JL: Pulmonary metastasectomy: a multivariate analysis of $\mathbf{4 4 0}$ patients undergoing complete resection. Interact Cardiovasc Thorac Surg 2012, 14(2):156-161.

\section{Figures}



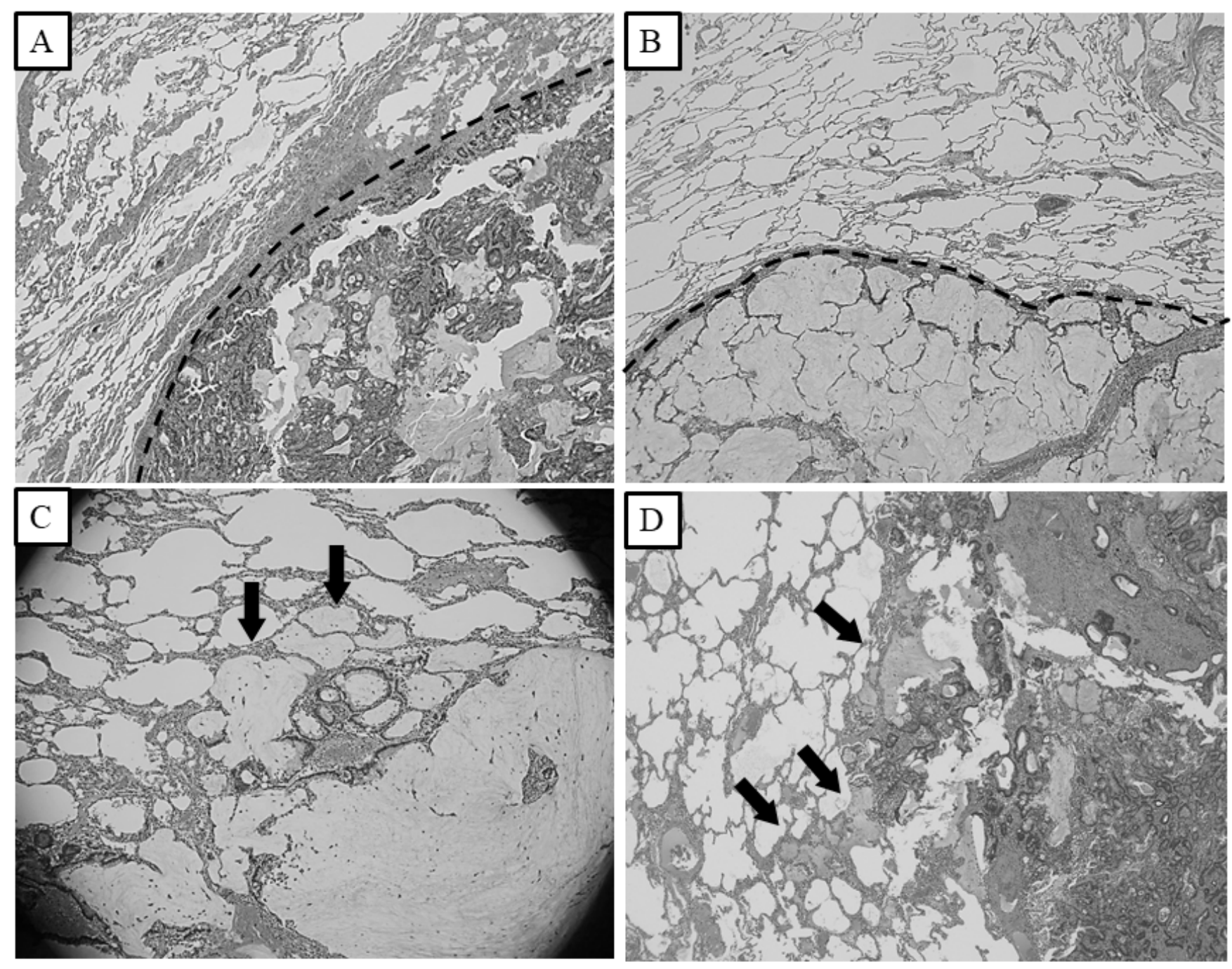

\section{Figure 1}

$(A, B)$ The border between the tumor/mucus and normal lung tissue is clear. A border is identified as an easily recognizable smooth surface, indicated here by a dotted line. (C, D) The border between the tumor/mucus and normal lung tissue is not clear. Mucus is identified within air spaces in the normal lung parenchyma beyond the edge of the tumor (arrows). Note: The designations employed and the presentation of the material on this map do not imply the expression of any opinion whatsoever on the part of Research Square concerning the legal status of any country, territory, city or area or of its authorities, or concerning the delimitation of its frontiers or boundaries. This map has been provided by the authors. 


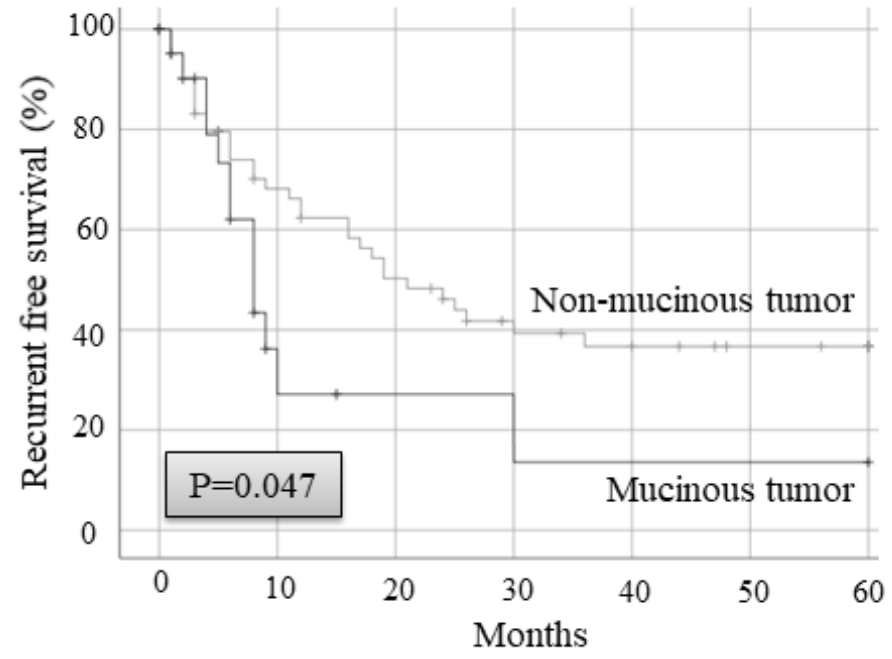

Figure 2

Recurrence-free survival after pulmonary resection in patients with mucinous tumors is $13.6 \%$, which is significantly lower than the $36.7 \%$ observed in patients with non-mucinous tumors. 


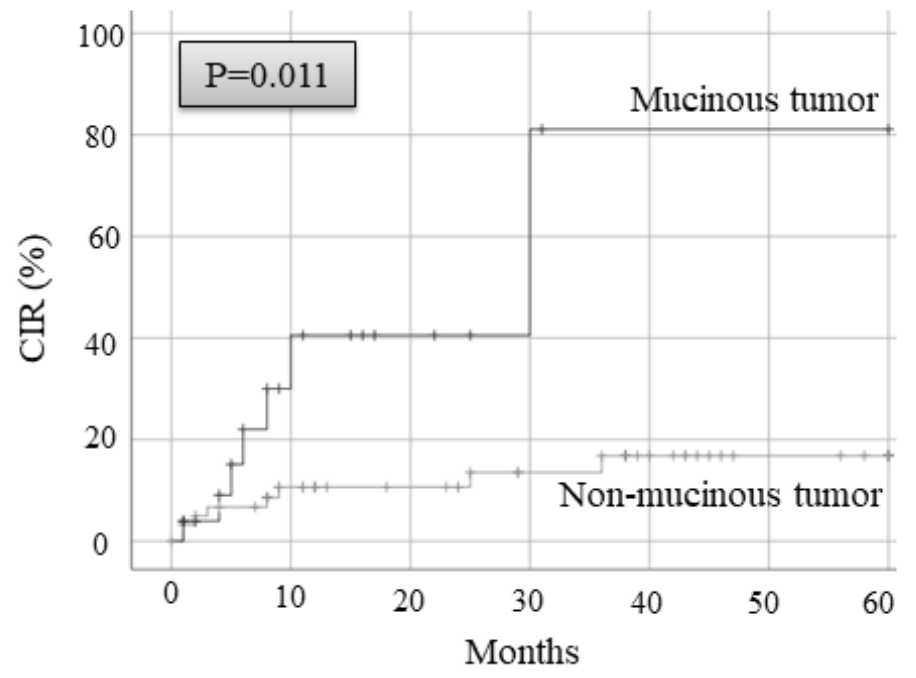

\section{Figure 3}

The 5-year CIR rate in patients with mucinous tumors is $80.3 \%$, which is significantly higher than the $15.5 \%$ observed in patients with non-mucinous tumors. CIR: cumulative incidence of locoregional recurrence. 


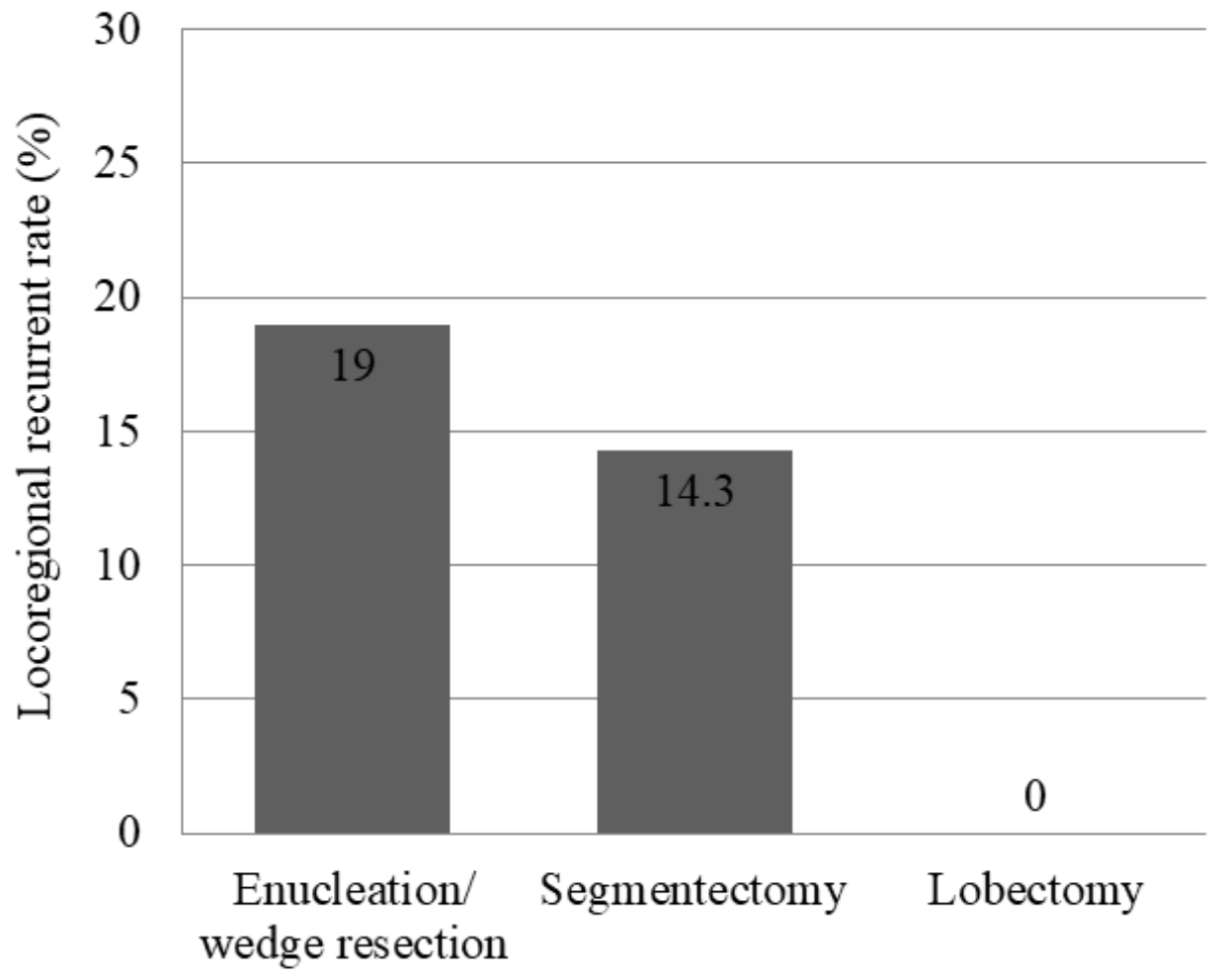

Figure 4

Locoregional recurrence rates are $19.0 \%$ for enucleation or wedge resection, $14.3 \%$ for segmentectomy, and $0 \%$ for lobectomy. 